\title{
Simulation of Spatial and Temporal Radiation Exposures for ISS in the South Atlantic Anomaly
}

\author{
Brooke M. Anderson \\ NASA Langley Research Center, Hampton, VA, 23681 \\ John E. Nealy \\ Old Dominion University, Norfolk, VA, 23508 \\ Nathan J. Luetke \\ Swales Aerospace, Newport News, VA, 23606 \\ Christopher A. Sandridge and Garry D. Qualls \\ NASA Langley Research Center, Hampton, VA 23681
}

The International Space Station (ISS) living areas receive the preponderance of ionizing radiation exposure from Galactic Cosmic Rays (GCR) and geomagnetically trapped protons. Practically all trapped proton exposure occurs when the ISS passes through the South Atlantic Anomaly (SAA) region. The fact that this region is in proximity to a trapping "mirror point" indicates that the proton flux is highly directional. The inherent shielding provided by the ISS structure is represented by a recently-developed CAD model of the current 11-A configuration. Using modeled environment and configuration, trapped proton exposures have been analytically estimated at selected target points within the Service and Lab Modules. The results indicate that the directional flux may lead to substantially different exposure characteristics than the more common analyses that assume an isotropic environment. Additionally, predictive capability of the computational procedure should allow sensitive validation with corresponding on-board directional dosimeters.

\section{Nomenclature}

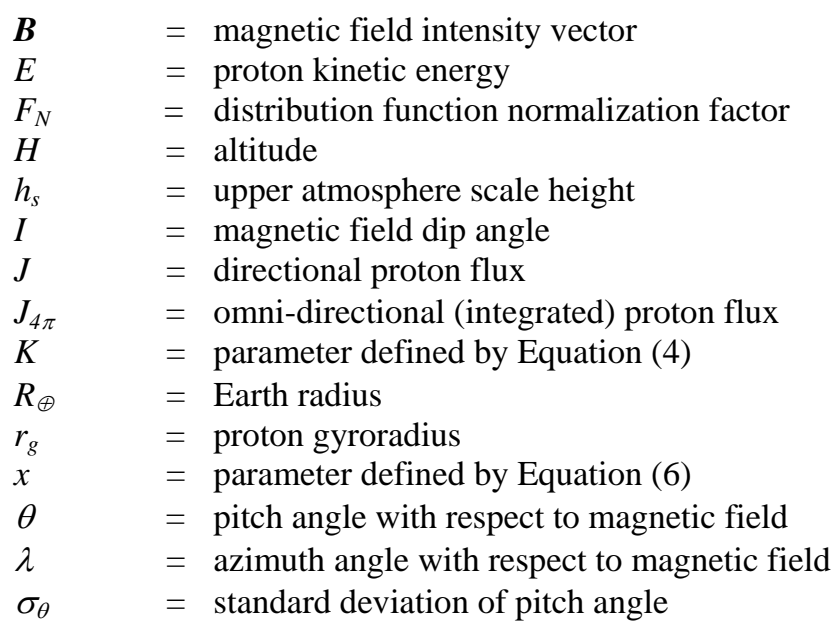




\section{Introduction}

The ISS at the present time has evolved as a near-Earth space habitat suitable for continuous human occupation. Further evolution of ISS should render it as facility forming a vital part of an expanding space exploration infrastructure. This study will look at the radiation exposure aspect of astronaut health and safety by utilizing analytical procedures for determining ionizing radiation dose with a view toward implementation as a means of shield augmentation for the habitation modules. A CAD model of the ISS 11-A configuration specifically dedicated to exposure analysis has been developed for this study.

The first step in the analytical process begins with establishment of an appropriate environment model. For the Low Earth Orbit (LEO) environment, the most important contributors to deposition of ionizing radiation energy are the trapped protons and the GCR. The present study addresses only the highly directional (vectorial) proton flux, which very roughly constitutes about half the total cumulative exposure for long duration missions. However, instantaneous dose rates are very much higher during the approximately 10 - 15 minute SAA transits for which most of the trapped proton exposure occurs during a 24-hour day. During the transits, both omni-directional and vector proton flux vary from near zero to maximum values, and directionality is controlled by the vehicle orientation with respect to the magnetic field vector components. Consequently, an added degree of complexity is introduced with the time variation of proton flux spectra along the orbit, for which individual transport properties through the shield medium must be taken into account. The deterministic high energy heavy ion transport code HZETRN ${ }^{1}$, developed at NASA-Langley, is used to describe the attenuation and interaction of the LEO environment particles along with the dosimetric quantities of interest. The ISS geometry defined by the CAD model is finally used to calculate exposures at selected target points within the modules, some of which represent locations of thermo-luminescent detectors (TLDs).

\section{LEO Environment and Proton Transport}

This section describes the radiation environment selected for the present study and its spatial variation in the SAA region. Nominal ISS orbital conditions are prescribed as $400 \mathrm{~km}$ altitude at 51.6-degree inclination. Simple circular orbit equations have been used to tailor the SAA transits for passage through peak flux regions. Time variation of the exposure is defined by these transits.

\section{A. SAA Protons for ISS Transit}

The standard NASA trapped proton model AP8MIN ${ }^{2}$ has been chosen to define a near-worst-case scenario for the fluxes. Fig. 1 depicts the orbital tracks in ascent and descent passing through the high flux regions.

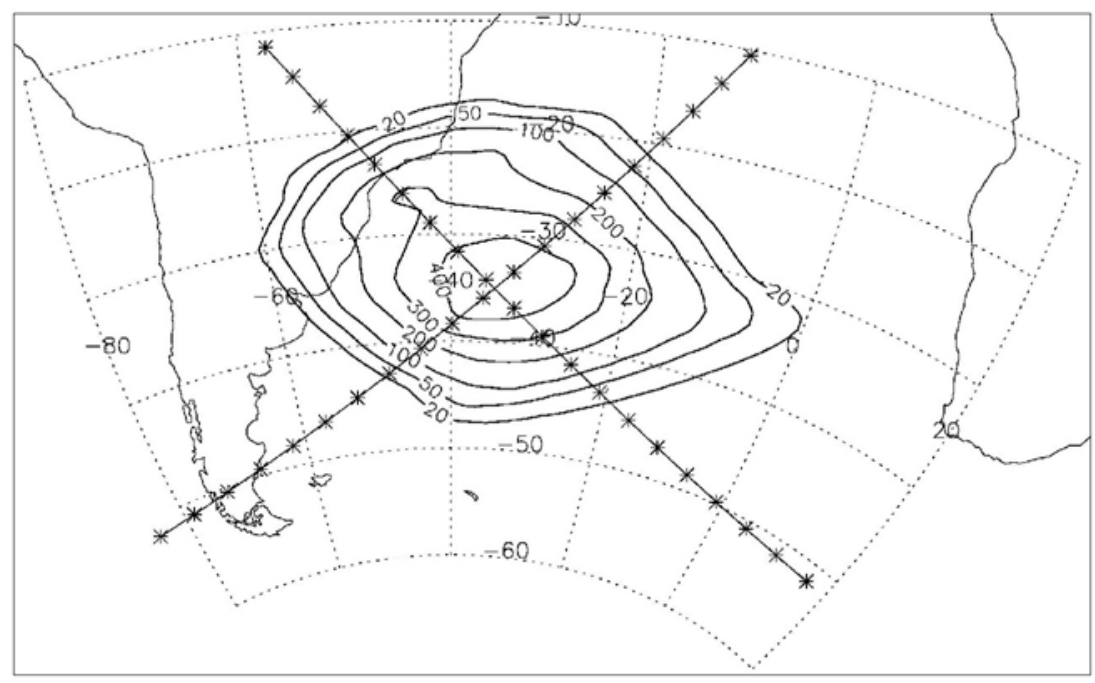

Figure 1. Ascent and descent orbital tracks for ISS through the South Atlantic Anomaly. Symbol spacing represents 1-min. intervals; flux contours are in units of protons $(>100 \mathrm{MeV}) / \mathrm{cm}^{2}$-sec. 
The differential flux spectra obtained from the environment model are plotted in Figs. 2a and 2b for selected points near the region of peak flux. The chosen points are identified by time values in minutes elapsed after ascending node point.
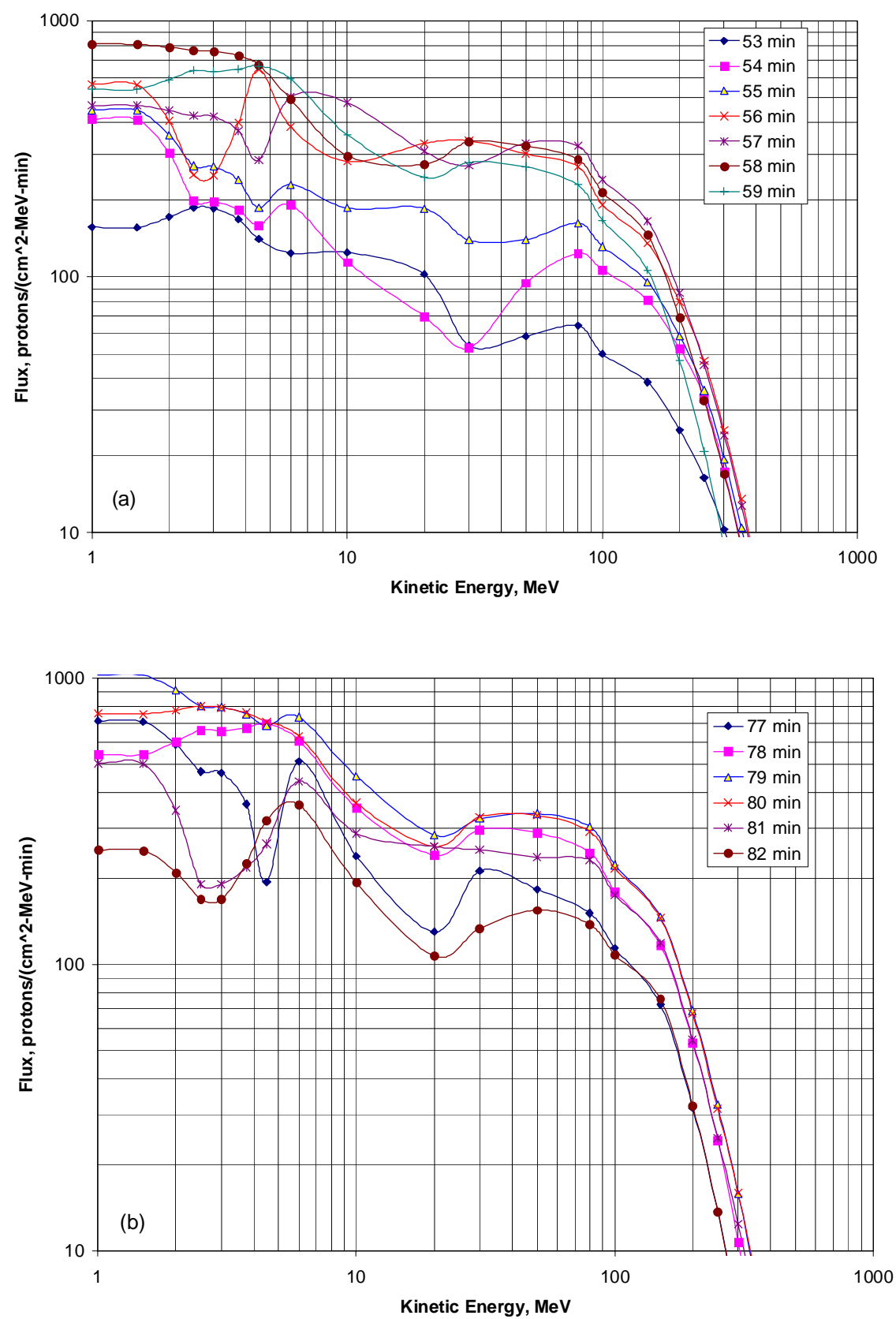

Figure 2. Omni-directional differential flux spectra obtained from the AP8MIN model in central region of SAA for (a) descending track and (b) ascending track. 
The complex low-energy behavior in the proton spectra is not readily explained and is most likely due to several influences. Since only higher energy protons ( $>\sim 50 \mathrm{MeV}$ ) penetrate the ISS structure, the low energy fluctuations are unimportant. In order to introduce directionality into the flux spectra, the local magnetic field properties become a major factor in the environment.

Near a mirror point, the spiraling particle paths are nearly normal to the field lines (i. e., pitch angle approaches $90^{\circ}$ ). A good account of the theoretical basis for the vector flux of protons in the SAA may be found in Heckman and $\mathrm{Nakano}^{3}$, and computational models have been developed for analyzing the effects of directionality ${ }^{4,5}$. Using critical assumptions and approximations, an expression for the directional flux has been found ${ }^{3}$ in terms of local magnetic field vector, $\mathbf{B}$; altitude, $\mathrm{H}$; ionospheric scale height, $h_{s}$, and the pitch and azimuth angles $(\theta$ and $\lambda)$. This formula, in the nomenclature of $\mathrm{Kern}^{5}$, is expressed as a ratio of the vector flux to the omnidirectional (integrated) value:

$$
\frac{J}{J_{4 \pi}}=F_{N} \exp \left[\frac{-(\pi / 2-\theta)^{2}}{2 \sigma_{\theta}^{2}}\right] \exp \left[\frac{r_{g} \cos I \cos \lambda}{h_{s}}\right]
$$

where $\mathrm{I}$ is the magnetic dip angle, and $\mathrm{r}_{\mathrm{g}}$ is the proton gyroradius given by

$$
r_{g}=\frac{\sin \theta \sqrt{E^{2}+1876 E}}{30|\vec{B}|}
$$

with the proton kinetic energy, $\mathrm{E}$, in $\mathrm{MeV}$ and magnetic field strength, $\mathrm{B}$, in gauss. The standard deviation of pitch angle is given by

$$
\sigma_{\theta}=\sqrt{\frac{h_{s}}{K \sin I}}
$$

where

$$
K=(4 / 3) \frac{R_{\oplus}+H}{\left(2+\cos ^{2} I\right) \sin I}
$$

with $\mathrm{R}_{\oplus}$ representing the earth radius. $\mathrm{F}_{\mathrm{N}}$ is a normalization factor, parameterized by $\mathrm{Kern}^{5}$ as:

$$
F_{N}=\left(.075 / \sigma_{\theta}\right)(.8533+x) \exp (-x)
$$

where

$$
x=\frac{r_{g} \cos I}{h_{s} \sin \theta}
$$

When the omni-directional flux is redistributed according to the distribution function of Equation (1), a pattern emerges in which most particles are directed in a very pronounced band of zenith and azimuth angles.

\section{B. Energetic Proton Transport in Shield Medium}

The spectra of Figure 2 have been used as input to the HZETRN code to compute transport through thickness ranges of shield material (Al). Subsequent exposures in simulated tissue $\left(\mathrm{H}_{2} \mathrm{O}\right)$ are evaluated as dose equivalents using ICRP ${ }^{6}$ quality factors for normally incident flux on semi-infinite slab geometry. The NASA-Langley HZETRN code is a well-established deterministic procedure allowing rapid and accurate solution to the Boltzmann transport equation. Details concerning the interaction and attenuation methodology are described at length elsewhere $^{1,7}$. Figures $3 a$ and $3 b$ show the resultant dose vs. depth functions obtained from the transport calculations that are used to evaluate ultimate exposures at target points within complex shield configurations defined by the desired CAD solid model of the full-scale geometric structure. 

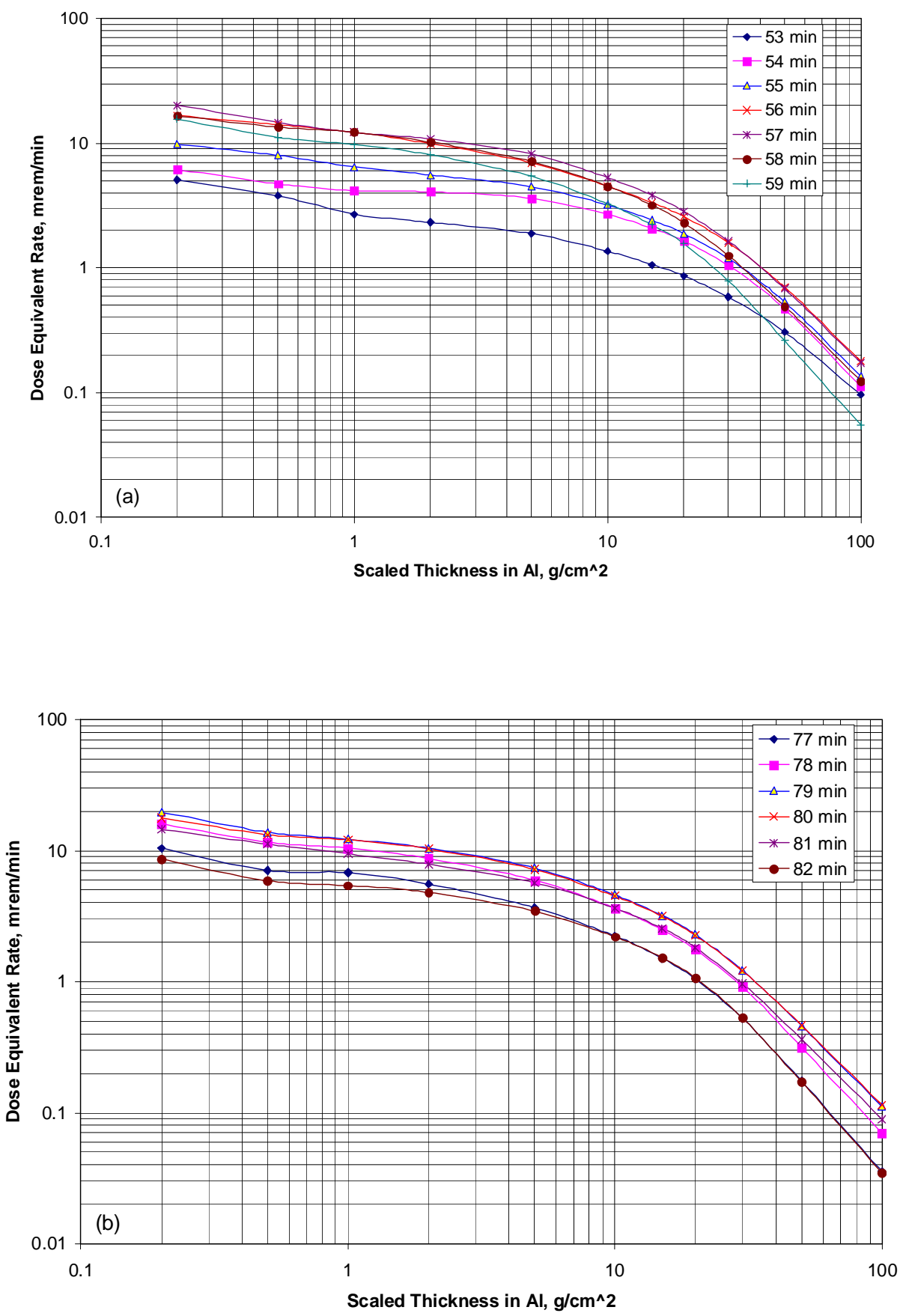

Figure 3. Dose vs. Depth functions calculated for Aluminum slab geometry at selected times during SAA transit: (a) descending track and (b) ascending track. 


\section{CAD Solid Model of ISS 11-A Configuration}

The primary components of the ISS 11-A configuration are the U. S. Destiny Lab Module, the U. S. Unity Connections Module (Node 1), the U. S. Airlock and the three U. S. Pressurized Mating Adaptors (PMAs). Also included are the Russian Functional Cargo Block (FGB, or Zarya), the Russian Service Module (SM, or Zvezda, the Russian Soyuz Spacecraft, the Russian Progress re-supply vehicle, the Russian Docking Compartment and the truss structures. A simplified model of this configuration has been constructed for dedicated shield analysis using the commercially available CAD software I-DEAS ${ }^{\circledR}$. This model consists of 460 separate components, each having its own dimensions, orientation, and density distribution defined in near conformity with the actual hardware. A large part of the inherent shielding for the astronauts results from the distributed micrometeoroid shield and the pressure vessel itself. The cargo in the primary modules also provides additional shielding. In this analysis it is assumed that these components are primarily made up of aluminum. A description of a predecessor (configuration 7-A) of the present model may be found in Hugger et al. ${ }^{8}$ Figures 4, 5, and 6 show an external view of the 11-A CAD model as it appears on a computer screen and split-view illustrations of the 6 target points chosen for this analysis.

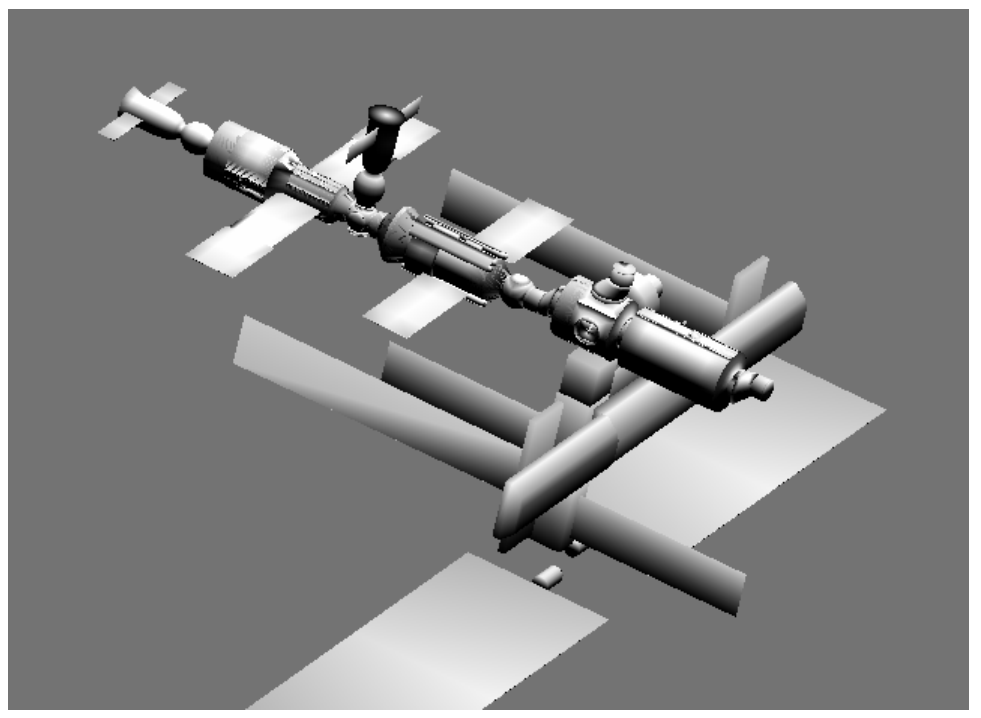

Figure 4. External perspective view of CAD Modeled ISS 11-A configuration.

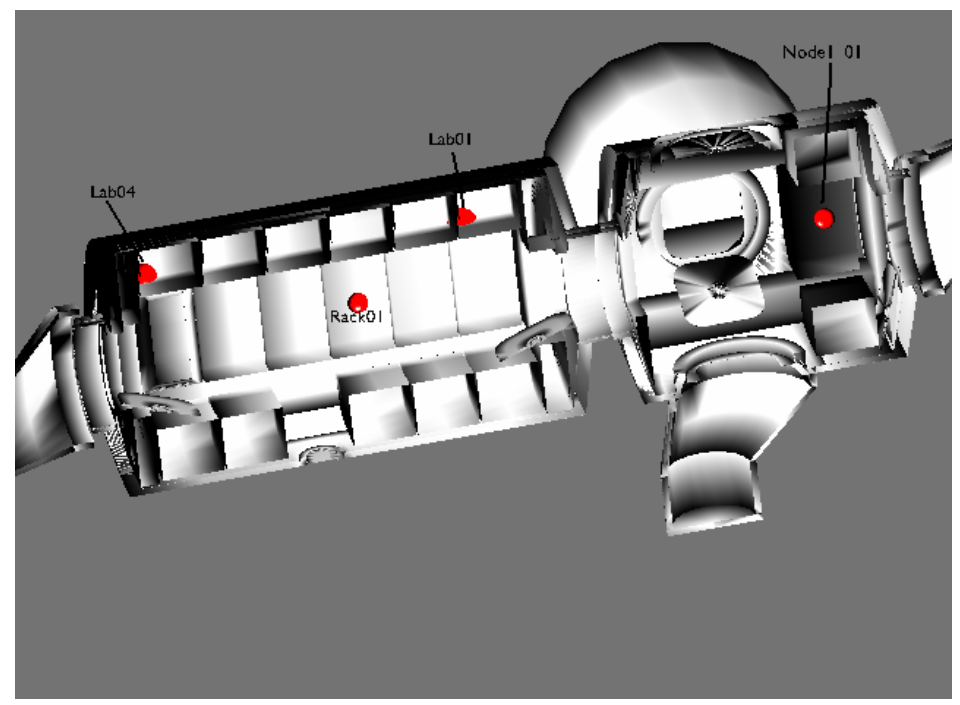

Figure 5. Split view of U. S. Lab Module showing selected target points. 


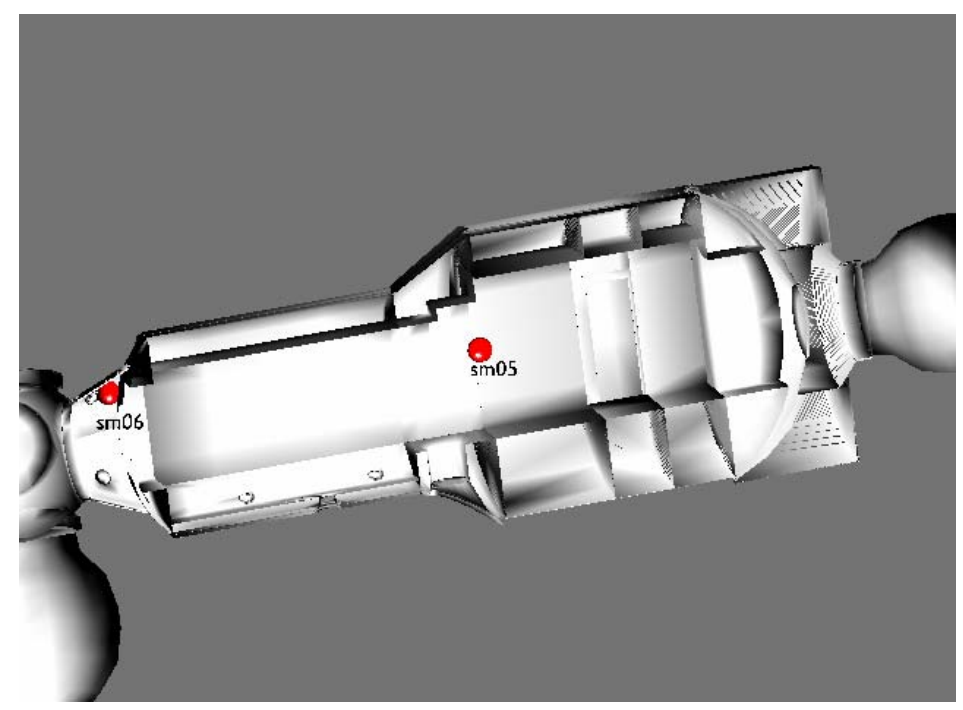

Figure 6. Depiction of selected target points in Russian Service Module.

The distributions of thickness for 970 directions has been evaluated in terms of the scaled thickness in $\mathrm{g} / \mathrm{cm}^{2}$ for each of the 6 chosen target points for a spherical coordinate system with origin at the point. The ray directions are determined for 22 polar angles and 44 azimuth angles plus 2 separate polar angles at top and bottom. The spherical coordinate grid is defined so that each directional ray subtends a constant solid angle. The cumulative distributions are given for the 6 points in Figure 7.

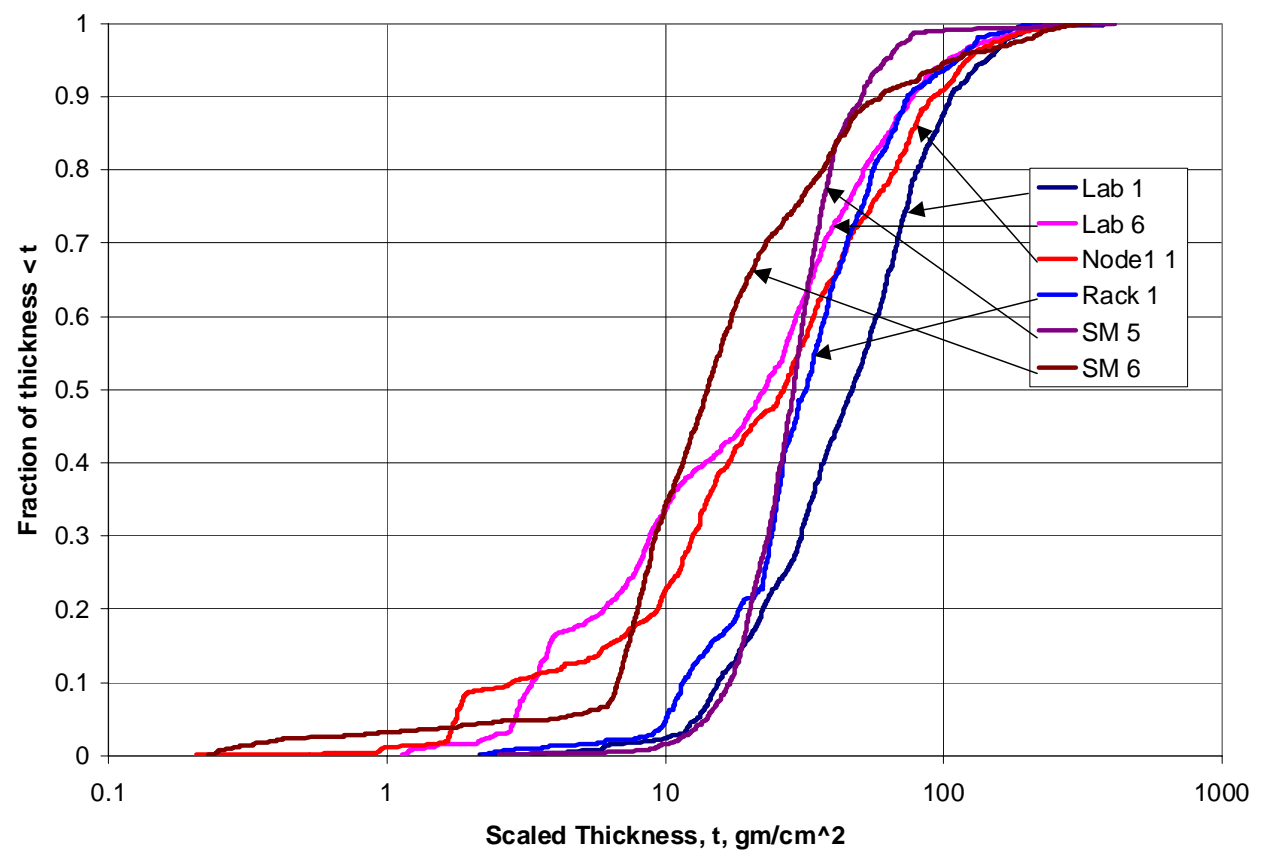

Figure 7. Cumulative thickness distribution for selected target points in ISS 11-A configuration. 


\section{Results of Calculations}

Table I. Calculated Dose Equivalent Rates (mrem/min) for Selected Target Points for Isotropic and Directional SAA Proton Environments

DESCENDING TRACK

\begin{tabular}{|c|c|c|c|c|c|c|}
\hline \multirow[b]{2}{*}{ Time Step } & \multicolumn{2}{|c|}{ RACK01 } & \multicolumn{2}{|c|}{ LAB1 } & \multicolumn{2}{|c|}{ LAB4 } \\
\hline & Directional & Omni & Directional & Omni & Directional & Omni \\
\hline 53 & 0.72 & 0.61 & 0.45 & 0.45 & 1.09 & 0.97 \\
\hline 54 & 1.29 & 1.11 & 0.67 & 0.79 & 1.99 & 1.80 \\
\hline 55 & 1.44 & 1.29 & 0.70 & 0.92 & 2.41 & 2.21 \\
\hline 56 & 1.87 & 1.78 & 0.92 & 1.27 & 3.63 & 3.36 \\
\hline 57 & 1.91 & 1.95 & 1.00 & 1.37 & 4.01 & 3.77 \\
\hline 58 & 1.43 & 1.58 & 0.84 & 1.10 & 3.40 & 3.27 \\
\hline 59 & 0.88 & 1.07 & 0.58 & 0.72 & 2.36 & 2.41 \\
\hline
\end{tabular}

NODE1_1

SM5

\begin{tabular}{|c|c|}
\hline \multicolumn{1}{|c|}{ Directional } & Omni \\
\hline 0.63 & 0.63 \\
\hline 1.11 & 1.16 \\
\hline 1.25 & 1.33 \\
\hline 1.66 & 1.80 \\
\hline 1.71 & 1.94 \\
\hline 1.28 & 1.54 \\
\hline 0.77 & 1.01 \\
\hline
\end{tabular}

SM6

\begin{tabular}{|c|c|}
\hline \multicolumn{1}{|c|}{ Directional } & Omni \\
\hline 1.26 & 1.18 \\
\hline 2.41 & 2.10 \\
\hline 2.89 & 2.55 \\
\hline 4.16 & 3.74 \\
\hline 4.66 & 4.55 \\
\hline 3.91 & 3.67 \\
\hline 2.83 & 2.98 \\
\hline
\end{tabular}

\section{ASCENDING TRACK}

\begin{tabular}{|c|c|c|c|c|c|c|}
\hline \multirow[b]{3}{*}{ Time Step } & \multicolumn{6}{|c|}{ 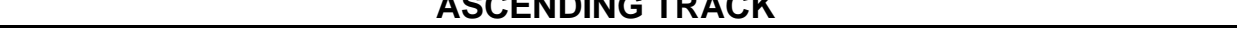 } \\
\hline & \multicolumn{2}{|c|}{ RACK01 } & \multicolumn{2}{|c|}{ LAB1 } & \multicolumn{2}{|c|}{ LAB4 } \\
\hline & Directional & Omni & Directional & Omni & Directional & Omni \\
\hline 77 & 0.67 & 0.72 & 0.41 & 0.49 & 1.17 & 1.64 \\
\hline 78 & 1.10 & 1.21 & 0.71 & 0.82 & 1.90 & 2.66 \\
\hline 79 & 1.41 & 1.57 & 0.95 & 1.08 & 2.39 & 3.32 \\
\hline 80 & 1.39 & 1.56 & 0.96 & 1.07 & 2.35 & 3.27 \\
\hline 81 & 1.10 & 1.24 & 0.77 & 0.86 & 1.86 & 2.58 \\
\hline 82 & 0.63 & 0.72 & 0.44 & 0.49 & 1.10 & 1.54 \\
\hline
\end{tabular}

NODE1_1

SM5

\begin{tabular}{c|c|c|} 
Time Step & Directional & Omni \\
\cline { 2 - 3 } 77 & 1.88 & 1.47 \\
\cline { 2 - 3 } 78 & 3.01 & 2.37 \\
\cline { 2 - 3 } 79 & 3.73 & 2.96 \\
80 & 3.66 & 2.92 \\
\cline { 2 - 3 } 81 & 2.89 & 2.30 \\
82 & 1.73 & 1.36 \\
\cline { 2 - 3 } & &
\end{tabular}

\begin{tabular}{|c|c|}
\hline \multicolumn{1}{|c|}{ Directional } & Omni \\
\hline 0.72 & 0.68 \\
\hline 1.19 & 1.16 \\
\hline 1.55 & 1.52 \\
\hline 1.51 & 1.51 \\
\hline 1.20 & 1.21 \\
\hline 0.68 & 0.69 \\
\hline
\end{tabular}

SM6

\begin{tabular}{|c|c|}
\hline \multicolumn{1}{|c|}{ Directional } & Omni \\
\hline 1.82 & 2.15 \\
\hline 2.98 & 3.20 \\
\hline 3.74 & 4.13 \\
\hline 3.61 & 3.81 \\
\hline 2.91 & 3.01 \\
\hline 1.68 & 1.97 \\
\hline
\end{tabular}


Each of the entries in the preceding table represents the solid-angle integration of dose equivalent rate resulting from protons incident on the target point from all directions. Even though the total doses are of the same magnitude for both isotropic and vectorial external environments, the directional properties of the radiation field may be vastly different for the two cases. This is illustrated in Fig. 8 for the target point designated RACK01 as spherical coordinate angle contour plots of the directional dose.
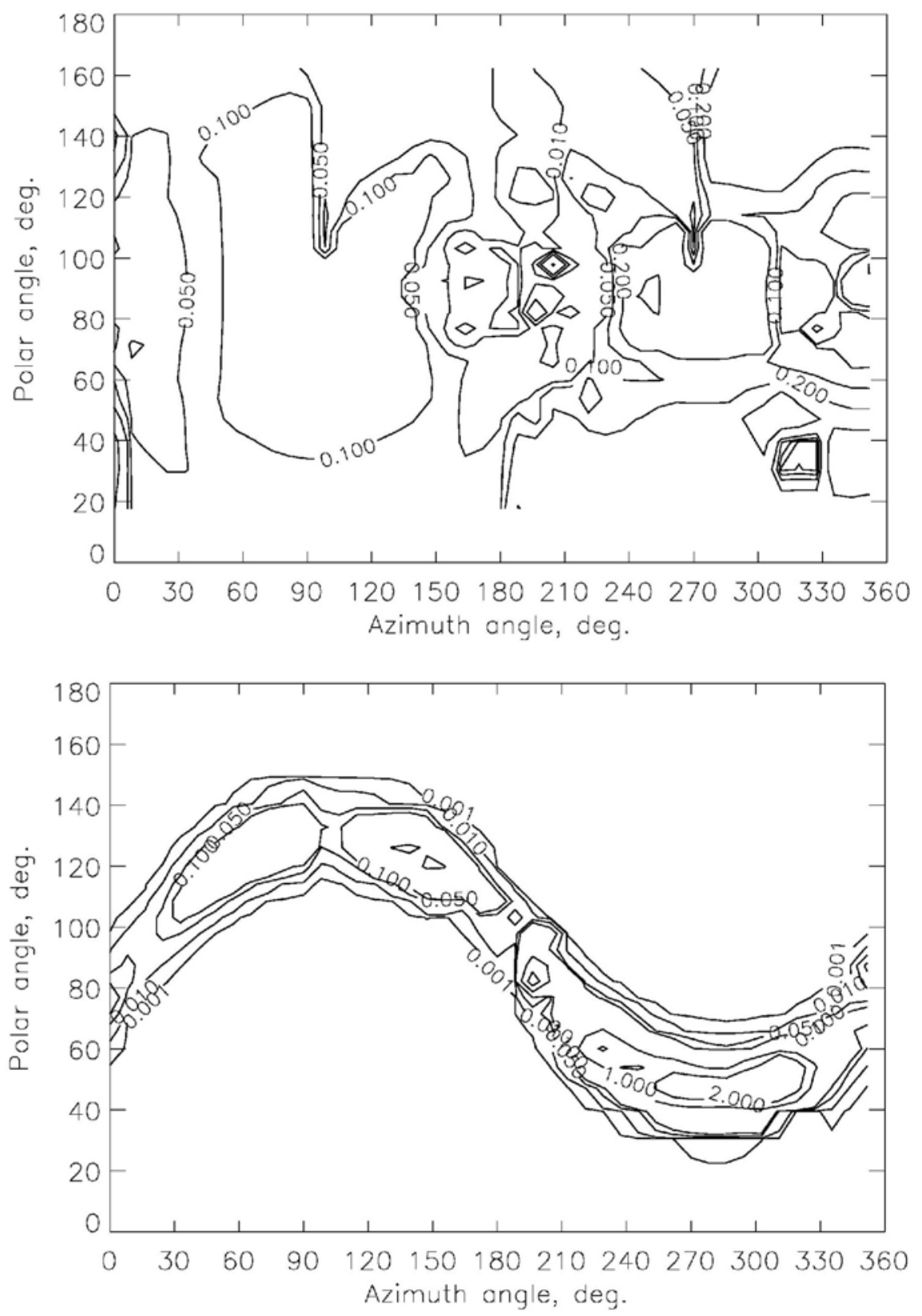

Figure 8. Contour plots of directional dose equivalent as functions of spherical coordinate angles about target point RACK01 for isotropic environment (top) and directional environment (bottom). Units are in $\mathrm{mrem} /(\mathbf{m i n}-\mathrm{sr})$. 


\section{Analysis of Results}

The contour maps of Fig. 8 portray the differences in directional dose distribution and illustrate quantitavely the angular variation of exposure intensity. However, such renditions are difficult to interpret and diagnose analytically. Present 3-D computer graphic visualization techniques may be implemented to provide displays that lend themselves to much more convenient and rapid interpretation.
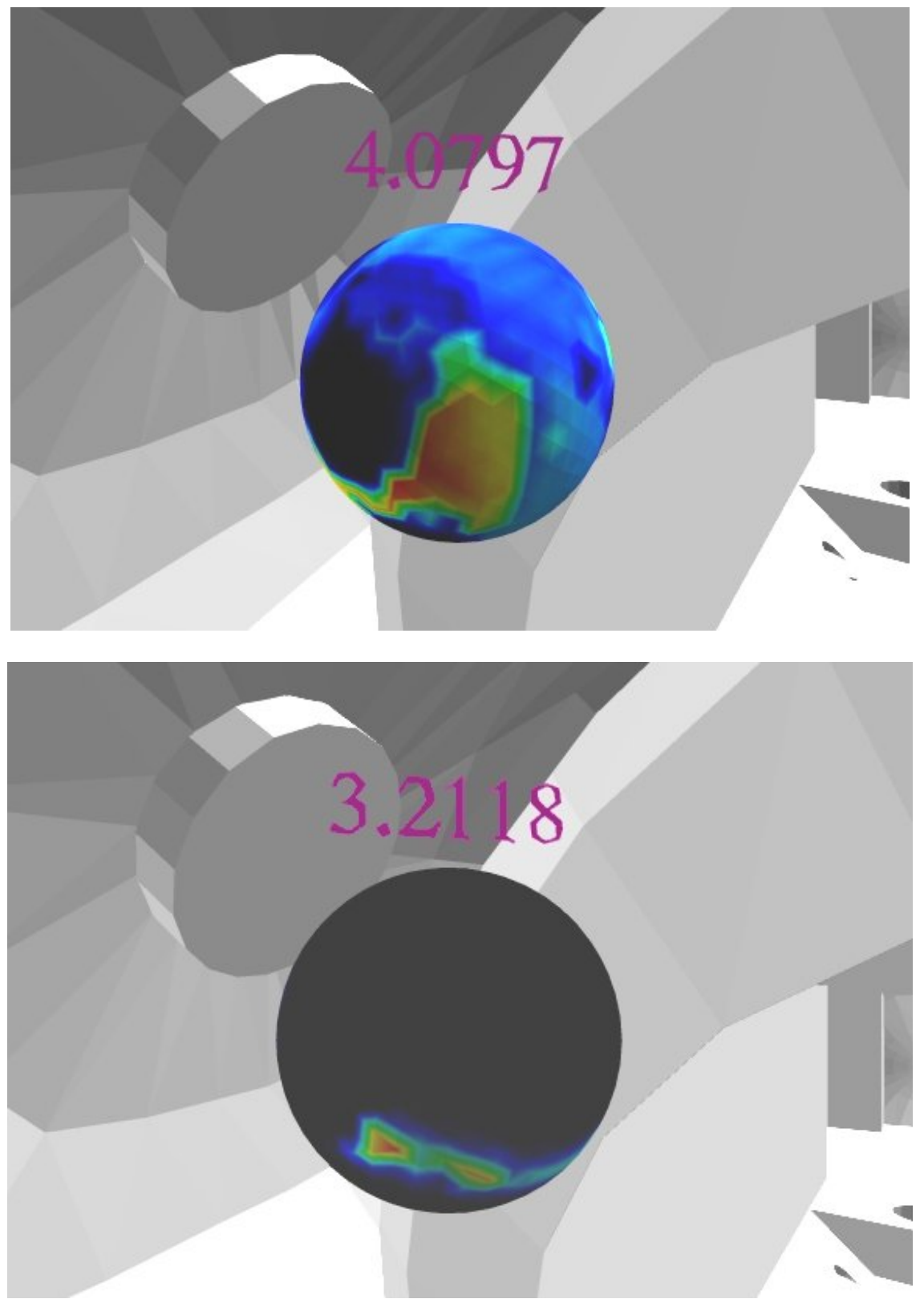

Figure 9. Computer-generated distributions of dose equivalent on spherical surfaces centered on target point within ISS CAD model for isotropic environment (top) and directional environment (bottom). 
The illustrations shown in Fig. 9 represent the application of visualization software exhibiting color-coded patterns of directional dose mapped onto a spherical surface. The example chosen is a point near that designated as SM6 in a relatively lightly-shielded region of the Service Module. The mapping is for a time step on the ascent path and demonstrates a case for which the isotropic and directional doses contrast markedly. Such images clearly show the impact of the normalized distribution function that results in a re-direction, or "focusing" of the isotropic flux. Consequently, in some cases the integrated dose in the directional case may be substantially less than for that of the isotropic environment. In other cases, the reverse may occur as may be seen in the tabular results. Such variations arise because of the complex interactions of the charged particle environment with the local magnetic field and the changing orientation of the vehicle structure.

\section{Summary and Conclusion}

The primary purpose of this study is to demonstrate by realistic simulation a procedure for accurately analyzing and predicting radiation exposures in the confines of a shielded spacecraft. The procedure described can readily be implemented in comprehensive specific mission analyses and shield design efforts. In the present study, we have attempted only to portray results pertaining to the exposures encountered by ISS in transit through the higher flux regions of SAA. A more detailed analysis along these lines would necessarily address the more realistic 2 or 3 SAA transits per day of ISS over an extended time period. Near-term plans are to progress from spatial/temporal simulation to real-time analyses as directional dosimeter data becomes available from ISS. Such validations will provide a stringent test of the adequacy of the theoretical developments and serve to quantify the predictive capabilities as they may apply to future human missions as well as to remote sensing platforms.

\section{References}

${ }^{1}$ Wilson, J. W., et al., "HZETRN: Description of a Free-Space Ion and Nucleon Transport and Shielding Computer Program,” NASA TP-3495, May 1995.

${ }^{2}$ Sawyer, D. M. and Vette, J. J., “AP-8 Trapped Proton Environments for Solar Maximum and Solar Minimum,” NSSDC WDC-A-R85 76-06, 1976.

${ }^{3}$ Heckman, H. H. and Nakano, G. H., “Low-Altitude Trapped Protons during Solar Minimum Period,” J. Geophys. Res. Space Physics, Vol. 74, No. 14, July 1969, pp. 3575-3590.

${ }^{4}$ Watts, J., Parnell, T., and Heckman, H. H., “Approximate Angular Distribution and Spectra for Geomagnetically Trapped Protons in Low Earth Orbit,” AIP Conference Proceedings on High Energy Radiation in Space, eds. Rester, A. C. and Trombka, J. I., Sanibel Is., FL, 1989, pp. 75-85.

${ }^{5}$ Kern, J. W., “A Note on Vector Flux Models for Radiation Dose Calculations,” Radiation Meas., Vol. 23, No. 1, 1994, pp. 75-85.

${ }^{6} 1990$ Recommendations of the International Commission on Radiological Protection, ICRP Publication 60, Annals of the ICRP, Vol. 21, Elsevier Science, N. Y., 1991.

${ }^{7}$ Wilson, J. W., et al., “Transport Methods and Interactions for Space Radiations,” NASA RP-1257, Dec. 1991.

${ }^{8}$ Hugger, C. P., et al., "Preliminary Validation of an ISS Radiation Shielding Model," Proceedings of AIAA Space 2003 Conference, AIAA Paper No. 2003-6220, Long Beach, CA, 23-25 Sept. 2003. 